\title{
Implicit Runge-Kutta Processes
}

\author{
By J. C. Butcher
}

1. Introduction. A Runge-Kutta process is a means of obtaining an approximation $\hat{y}$ to the solution at $x=x_{0}+h$ for the system

$$
\frac{d \mathbf{y}}{d x}=\mathbf{f}(\mathbf{y}), \quad \mathrm{y}=\mathrm{y}_{0} \quad \text { at } \quad x=x_{0},
$$

where $\mathrm{y}$ is a vector of $n$ elements and $\mathbf{f}(\mathbf{y})$ a vector function of these elements.

The equations defining $\hat{y}$ for a $\nu$ stage Runge-Kutta process are*

$$
\begin{aligned}
\mathbf{g}^{(i)} & =\mathbf{f}\left(\mathrm{y}_{0}+h \sum_{j=1}^{\nu} a_{i j} \mathbf{g}^{(j)}\right) \quad(i=1,2, \cdots, \nu), \\
\hat{\mathbf{y}} & =\mathrm{y}_{0}+h \sum_{i=1}^{\nu} b_{i} \mathbf{g}^{(i)},
\end{aligned}
$$

where the coefficients $a_{i j}, b_{i}(i, j=1,2, \cdots, \nu)$ are numerical constants.

It was shown [1] that the true solution $y$ and the approximation $\hat{\mathrm{y}}$ can be expanded in power series given by the equations $\dagger$

$$
\begin{gathered}
\mathrm{y}=\mathrm{y}_{0}+\sum \alpha \mathrm{F} \frac{h^{r}}{r !}, \\
\hat{\mathrm{y}}=\mathrm{y}_{0}+\sum \beta \Phi \mathrm{F} \frac{h^{r}}{(r-1) !} .
\end{gathered}
$$

The summations are over the different "elementary differentials" $\mathbf{F}$ for the function $\mathbf{f}$, arranged in a sequence of non-decreasing $r$, the order of $\mathbf{F}$. $\Phi$ is the corresponding "elementary weight" and $\alpha, \beta$ are numerical coefficients independent of the form of $f$. Some formulae and tables for $\alpha$ and $\beta$ are given in [1].

Thus if $\mathbf{y}, \hat{\mathbf{y}}$ are to agree to terms in $h^{\rho}$ we must have

$$
\Phi=\frac{1}{\gamma}
$$

where $\gamma=r \beta / \alpha$, for all $\Phi$ for which $r \leqq \rho$.

A general Runge-Kutta process will be called "implicit" in contrast to those processes in which $a_{i j}=0$ for $i<j$; these will be called "semi-explicit." If in addition $a_{i j}=0$ when $i=j$ the process will be called "explicit." It has been traditional (for example $[2,3,4,5,6,7]$ ) to consider only explicit processes.

Received November 1, 1962. Revised April 22, 1963.

* If the function $\mathbf{f}(\mathbf{y})$ satisfies a Lipschitz condition and $h$ is sufficiently small, then the equations defining $\mathbf{g}^{(1)}, \mathbf{g}^{(2)}, \cdots, \mathbf{g}^{(v)}$ have a unique solution which may be found by iteration (see Appendix).

$\dagger$ It will be assumed throughout that $f(y)$ and all its derivatives exist and are continuous so that the Taylor expansions for $\mathbf{y}$ and $\hat{\mathbf{y}}$ may be terminated at any term with an error of the same order as the first term omitted. 
For convenience we shall designate the process by an array as follows

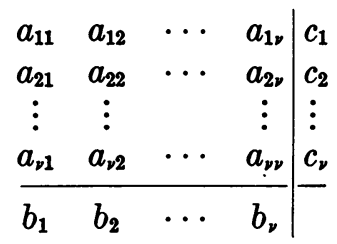

where $c_{i}=\sum_{j=1}^{\nu} a_{i j}$.

A well known example of an explicit process is the following due to Kutta [3]; in this case $\rho=3$.

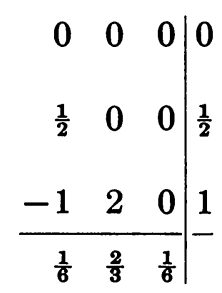

In contrast we have as examples of implicit and of semi-explicit processes the following:
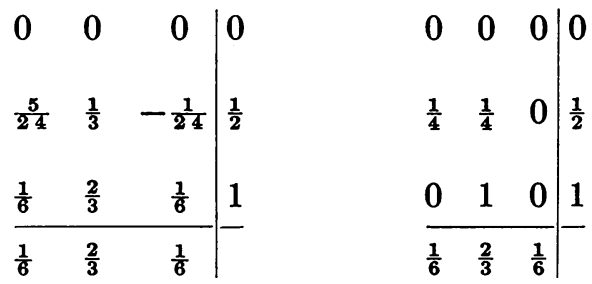

The first of these is equivalent to a process due to R. F. Clippinger and B. Dimsdale and quoted by Kunz [8]. On the other hand the semi-explicit process appears to have been previously overlooked even though it is of comparable accuracy and more convenient for practical use. Using results proved in section 2 of this paper or by simply verifying (3) for the appropriate $\Phi$ it is easy to verify that $\rho=4$ in each case.

It is well to consider what we might hope to gain by relaxing the restriction of allowing only explicit processes. In explicit processes we have $\nu(\nu+1) / 2\left(=N_{E}\right.$, say) coefficients $a_{i j}, b_{i}$ to choose while in the semi-explicit case the number is increased to $N_{\mathrm{s}}=\nu(\nu+3) / 2$. However, for implicit processes we have available the complete set of $N_{\mathrm{I}}=\nu(\nu+1)$ coefficients. It is reasonable to hope that with $N$ variables we can satisfy $M$ restrictions as long as $M \leqq N$.

The numbers $N_{\mathrm{E}}, N_{\mathrm{S}}, N_{\mathrm{I}}$ are shown in Table 1 as are the numbers of restrictions $M$ corresponding to different values of $\rho$. This number is, of course, the number of elementary differentials with orders not exceeding $\rho$, or what is equivalent, the number of rooted trees with no more than $\rho$ nodes [9].

Basing our comparison on this table we see that the gain in accuracy that can be achieved by the use of implicit rather than explicit processes is not more than one power of $h$ and in some cases semi-explicit processes do not give even this gain. 
TABLE 1

\begin{tabular}{r|rrr||l|r}
$\nu$ & $N_{\mathrm{E}}$ & $N_{\mathrm{S}}$ & $N_{\mathrm{I}}$ & $\rho$ & $M$ \\
1 & 1 & 2 & 2 & 1 & 1 \\
2 & 3 & 5 & 6 & 2 & 2 \\
3 & 6 & 9 & 12 & 3 & 4 \\
4 & 10 & 14 & 20 & 4 & 8 \\
5 & 15 & 20 & 30 & 5 & 17 \\
6 & 21 & 27 & 42 & 6 & 37 \\
7 & 28 & 35 & 56 & 7 & 85
\end{tabular}

However, the type of argument based on merely counting the number of equations of the form (3) that must be satisfied, ignores the relationships between them. In fact, it happens that the $M$ restrictions of Table 1 can often be satisfied with considerably fewer than $M$ variables.

Section 2 of this paper will be devoted to some general results on the relationships between the different $\Phi$, and the following sections will contain a study of a set of processes which are generalizations of the Gauss-Legendre quadrature formulae.

2. Some General Results. It is convenient to restrict ourselves in this paper to processes in which $c_{1}, c_{2}, \cdots, c_{\nu}$ are all distinct and none of $b_{1}, b_{2}, \cdots, b_{\nu}$ vanishes. If these restrictions are relaxed some results of this section will require slight modifications.

We shall use the symbols $A, B, C, D, E$ to represent certain statements about the numbers $a_{i j}, b_{i}$. These statements will depend on one or, in the case of $E$, two integral parameters which we will write as though they were arguments and the statement symbol a function of them. In the definitions of the symbols which now follow, $k$ and $l$ will always denote positive integers.

$$
\begin{aligned}
& A(\xi): \quad \Phi=\frac{1}{\gamma} \text { whenever } r \leqq \xi, \\
& B(\xi): \quad\left[\phi^{k-1}\right] \equiv \sum_{i=1}^{\nu} b_{i} c_{i}^{k-1}=\frac{1}{k} \text { for } k \leqq \xi, \\
& C(\xi): \quad \sum_{j=1}^{\nu} a_{i j} c_{j}^{k-1}=\frac{c_{i}^{k}}{k} \text { for } i=1,2, \cdots, \nu \text { and } k \leqq \xi, \\
& D(\xi): \quad \sum_{i=1}^{\nu} b_{i} c_{i}^{k-1} a_{i j}=\frac{b_{j}\left(1-c_{j}^{k}\right)}{k} \text { for } j=1,2, \cdots, \nu \text { and } k \leqq \xi, \\
& E(\xi, \eta):\left[\phi^{k-1}\left[\phi^{l-1}\right]\right] \equiv \sum_{i=1}^{\nu} \sum_{j=1}^{\nu} b_{i} c_{i}^{k-1} a_{i j} c_{j}^{l-1}=\frac{1}{l(k+l)} \quad \text { for } k \leqq \xi \text { and } l \leqq \eta .
\end{aligned}
$$

A number of theorems which are needed in the later sections of this paper can be expressed conveniently in terms of these symbols. After a statement of these the proofs will follow.

TheOREM 1. If $A(\xi)$, then $B(\xi)$.

Theorem 2. If $A(\xi+\eta)$, then $E(\xi, \eta)$. 
Theorem 3. If $B(\xi+\eta)$ and $C(\eta)$, then $E(\xi, \eta)$.

Theorem 4. If $B(\xi+\eta)$ and $D(\xi)$, then $E(\xi, \eta)$.

Theorem 5. If $B(\nu+\eta)$ and $E(\nu, \eta)$, then $C(\eta)$.

Theorem 6. If $B(\xi+\nu)$ and $E(\xi, \nu)$, then $D(\xi)$.

Theorem 7. If $B(\zeta), C(\eta)$ and $D(\xi)$, where $\zeta \leqq \xi+\eta+1, \zeta \leqq 2 \eta+2$, then $A(\zeta)$.

To prove Theorem 1, we note that $r=k$ for $\Phi=\left[\phi^{k-1}\right]$. Also from equation (31) of [1] we see that $\gamma=k$. Similarly for $\Phi=\left[\phi^{k-1}\left[\phi^{l-1}\right]\right]$ we find $r=k+l$, $\gamma=l(k+l)$ so Theorem 2 follows.

Theorem 3 may be verified immediately. If $k \leqq \xi, l \leqq \eta$ then of the three expressions

$$
\begin{gathered}
\sum_{i=1}^{\nu} \sum_{j=1}^{\nu} b_{i} c_{i}^{k-1} a_{i j} c_{j}^{l-1}, \\
\frac{1}{l} \sum_{i=1}^{\nu} b_{i} c_{i}^{l+k-1}, \\
\frac{1}{l(l+k)},
\end{gathered}
$$

(4) and (5) are equal by $C(\eta)$ whereas (5) and (6) are equal by $B(\xi+\eta)$.

Theorem 5 is a sort of converse to theorem 3. If $k \leqq \nu, l \leqq \eta(5)$ and (6) are equal by $B(\nu+\eta)$ while (4) and (6) are equal by $E(\nu, \eta)$. Hence we have

$$
\sum_{i=1}^{\nu} b_{i} c_{i}^{k-1}\left(\sum_{j=1}^{\nu} a_{i j} c_{j}^{l-1}-\frac{1}{l} c_{i}^{l}\right)=0 \text {. }
$$

For $k=1,2, \cdots, \nu$ this constitutes a set of $\nu$ homogeneous linear equations in the $\nu$ variables $\left(\sum_{j=1}^{\nu} a_{i j} c_{j}^{l-1}-\Gamma^{-1} c_{i}^{l}\right), i=1,2, \cdots, \nu$. Since the matrix of coefficients is non singular, these variables all vanish, a result equivalent to Theorem 5.

To prove Theorem 4 we see that if $k \leqq \xi, l \leqq \eta$ then (4) and (6) are each equal (by $D(\xi)$ and $B(\xi+\eta)$ respectively) to

$$
\frac{1}{k} \sum_{j=1}^{\nu}\left(b_{j} c_{j}^{l-1}-b_{j} c_{j}^{k+l-1}\right)
$$

so the result follows.

Theorem 6 bears the same sort of relationship to Theorem 4 as Theorem 5 bears to Theorem 3. If $k \leqq \xi, l \leqq \nu(6)$ and (7) are equal by $B(\xi+\nu)$ and (4) and (6) are equal by $E(\xi, \nu)$. Thus

$$
\sum_{j=1}^{\nu} c_{j}^{l-1}\left(\sum_{i=1}^{\nu} b_{i} c_{i}^{k-1} a_{i j}-\frac{1}{k} b_{j}\left(1-c_{j}^{k}\right)\right)=0
$$

for $l=1,2, \cdots, \nu$ and the result follows in the same way as for Theorem 5 .

Finally, assuming that $\zeta \leqq \xi+\eta+1, \zeta \leqq 2 \eta+2$ we will prove Theorem 7 , that $A(\zeta)$ follows from $B(\zeta), C(\eta)$ and $D(\xi)$. We shall use the symbol $\equiv$ to denote the identity of two expressions when regarded as linear functions of $b_{1}, b_{2}, \cdots, b_{v}$. Thus if $\Phi=\sum_{i=1}^{i} b_{i} \chi_{i}, \Phi^{\prime}=\sum_{i=1}^{\prime} b_{i} \chi_{i}^{\prime}$ where $\chi_{i}, \chi_{i}^{\prime}$ are functions of $a_{11}, a_{12}, \cdots, a_{v v}$ then $\Phi \equiv \Phi^{\prime}$ will mean $\chi_{i}=\chi_{i}^{\prime}, i=1,2, \cdots, \nu$. 
Using this symbol we see immediately that if $\Phi_{i}=\Phi_{i}{ }^{\prime}(i=1,2, \cdots, s)$ then $\left[\Phi_{1} \Phi_{2} \cdots \Phi_{8}\right] \equiv\left[\Phi_{1}{ }^{\prime} \Phi_{2}{ }^{\prime} \cdots \Phi_{s}{ }^{\prime}\right]$. As a preliminary to the proof of the theorem we show by induction the lemma that

$$
\gamma \Phi \equiv r\left[\phi^{r-1}\right]
$$

when $r \leqq \eta+1$. If we suppose $\Phi=\left[\Phi_{1} \Phi_{2} \cdots \Phi_{8}\right]$ and that

$$
\gamma_{i} \Phi_{i} \equiv r_{i}\left[\phi^{r_{i}-1}\right] \dagger
$$

since the orders $r_{1}, r_{2} \cdots r_{s}$ of $\Phi_{1}, \Phi_{2} \cdots, \Phi_{s}$ are each less than $r$, we have

$$
\gamma=r \gamma_{1} \gamma_{2} \cdots \gamma_{s}
$$

so that

$$
\begin{aligned}
\gamma \Phi & \equiv r \gamma_{1} \gamma_{2} \cdots \gamma_{8}\left[\Phi_{1} \Phi_{2} \cdots \Phi_{8}\right] \\
& \equiv r r_{1} r_{2} \cdots r_{s}\left[\left[\phi^{r_{1}-1}\right]\left[\phi^{r_{2}-1}\right] \cdots\left[\phi^{r_{s}-1}\right]\right] \\
& \equiv r \sum_{i=1}^{\nu}\left\{b_{i}\left(r_{1} \sum_{j=1}^{\nu} a_{i j} c_{j}^{r_{1}-1}\right)\left(r_{2} \sum_{j=1}^{\nu} a_{i j} c_{j}^{r_{2}-1}\right) \cdots\left(r_{s} \sum_{j=1}^{\nu} a_{i j} c_{j}^{r_{s}-1}\right)\right\}
\end{aligned}
$$

Using $C(\eta)$ and that fact that none of $r_{1}, r_{2} \cdots, r_{s}$ can exceed $\eta$ we find

$$
\begin{aligned}
\gamma \Phi & \equiv r \sum_{i=1}^{\nu} b_{i} c_{i}^{r_{1}+r_{2}+\cdots+r_{s}} \\
& \equiv r\left[\phi^{r-1}\right]
\end{aligned}
$$

proving the lemma.

In this result we may now replace $\equiv$ by $=$ and, by $B(\zeta)$ and the lemma we have $A(\eta+1)$. To prove the result of the theorem for $\zeta \geqq r>\eta+1$ we write $\Phi=\left[\Phi_{1} \Phi_{2} \cdots \Phi_{s}\right]$ and suppose that $\Phi^{\prime}$ is one of $\Phi_{1}, \Phi_{2}, \cdots, \Phi_{s}$ chosen so that its order $r^{\prime}$ is not exceeded by any of $r_{1}, r_{2}, \cdots, r_{s}$. For $r^{\prime} \leqq \eta$ the lemma still holds so we need consider only the cases $r^{\prime}>\eta$. We now use induction on $r^{\prime}, r$ assuming the result true for all lower values of $r$ and with a given $r$ for all lower values of $r^{\prime}$.

No two of $r_{1}, r_{2}, \cdots, r_{s}$ can exceed $\eta$ for otherwise we would have

$$
r=1+r_{1}+r_{2}+\cdots+r_{s} \geqq 2 \eta+3>\zeta .
$$

Using the same sort of calculation as in the proof of the lemma we find

$$
\begin{aligned}
\gamma \Phi & \equiv r \gamma^{\prime}\left[\Phi^{\prime} \phi^{r-r^{\prime}-1}\right] \\
& \equiv r \gamma^{\prime} \sum_{i=1}^{\nu} b_{i} c_{i}^{r-r^{\prime-1}} a_{i j} \chi_{j}^{\prime}
\end{aligned}
$$

where

$$
\Phi^{\prime} \equiv \sum_{i=1}^{\nu} b_{i} \chi_{i}^{\prime}
$$

$\dagger$ Throughout this paper the convention will be adopted that if an elementary differential is assigned a subscript, superscript or other distinguishing mark, the same marks will be used with the corresponding elementary weights and the numerical constants $\alpha, \beta, \gamma$. 
and, by the induction hypothesis,

$$
\Phi^{\prime}=\frac{1}{\gamma^{\prime}} .
$$

Now $r-r^{\prime} \leqq(\xi+\eta+1)-(\eta+1)=\xi$. Hence, using $D(\xi)$ we have

$$
\begin{aligned}
\gamma \Phi & =\frac{r \gamma^{\prime}}{r-r^{\prime}} \sum_{i=1}^{\nu} b_{i}\left(1-c_{i}^{r-r^{\prime}}\right) \chi_{i}^{\prime} \\
& =\frac{r \gamma^{\prime}}{r-r^{\prime}}\left(\Phi^{\prime}-\left[\phi^{r-r} \Phi_{1}^{\prime} \Phi_{2}^{\prime} \cdots \Phi_{s^{\prime}}^{\prime}\right]\right)
\end{aligned}
$$

where $\Phi^{\prime}$ being of order greater than 1 can be written

$$
\Phi^{\prime} \equiv\left[\Phi_{1}^{\prime} \Phi_{2}^{\prime} \cdots \Phi_{8^{\prime}}^{\prime}\right] \text {. }
$$

The orders of $\Phi_{1}{ }^{\prime}, \Phi_{2}{ }^{\prime}, \cdots, \Phi_{8^{\prime}}^{\prime}$ are each less than $r^{\prime}$ so, using the induction hypothesis, a short calculation enables us to evaluate

$$
\left[\phi^{r-r^{\prime}} \Phi_{1}^{\prime} \Phi_{2}^{\prime} \cdots \Phi_{s^{\prime}}^{\prime}\right]=\frac{r^{\prime}}{\gamma^{\prime} r} .
$$

Hence

$$
\gamma \Phi=\frac{r \gamma^{\prime}}{r-r^{\prime}}\left(\frac{1}{\gamma^{\prime}}-\frac{r^{\prime}}{\gamma^{\prime} r}\right)=1
$$

proving Theorem 7 .

3. Integration Processes Based on Gaussian Quadrature Formulas. For the system

$$
\left.\begin{array}{ll}
\frac{d y_{1}}{d x}=f\left(y_{2}\right), & y_{1}=0 \\
\frac{d y_{2}}{d x}=1, & y_{2}=x_{0}
\end{array}\right\} \text { at } x=x_{0},
$$

the integration process reduces to a means of evaluating $I=\int_{x_{0}}^{x_{0}+h} f(x) d x$ using the quadrature formula $I=h \sum_{i=1}^{\nu} b_{i} f\left(x_{0}+h c_{i}\right)$. For this formula to be accurate to terms in $h^{\rho}$, it is not necessary for all the conditions implied by $A(\rho)$ to be satisfied but only those contained in the statement $B(\rho)$. Hence to each Runge-Kutta process there corresponds a quadrature formula characterized by the values of $b_{1}, b_{2}, \cdots, b_{\nu} ; c_{1}, c_{2}, \cdots, c_{\nu}$. In this section we concern ourselves with the well known Gauss-Legendre quadrature formulae [10]. It will be found that to each such formula, as adapted for integrating in the range $\left(x_{0}, x_{0}+h\right)$, there corresponds a unique Runge-Kutta process with the same order of accuracy.

For the form of the Gauss-Legendre formula that we use, $c_{1}, c_{2}, \cdots, c_{\nu}$ are the roots of the equation $P_{\nu}(2 c-1)=0$, where $P_{\nu}(x)$ is the Legendre polynomial of degree $\nu$. The consequence of this choice is that when any $\nu$ of the equations of the set $B(2 v)$ hold the rest do also so that $b_{1}, b_{2}, \cdots, b_{v}$ can be found as solutions to these equations.

The important results of this section are the following: 


$$
\begin{aligned}
& \nu=1: \quad \frac{1}{2}\left|\frac{1}{1}\right| \\
& \nu=2: \quad \begin{array}{ccc|c}
\frac{1}{4} & \frac{1}{4}-\frac{\sqrt{3}}{6} & \frac{1}{2}-\frac{\sqrt{3}}{6} \\
\hline \frac{1}{4}+\frac{\sqrt{3}}{6} & \frac{1}{4} & \frac{1}{2}+\frac{\sqrt{3}}{6} \\
\hline \frac{1}{2} & \frac{1}{2} &
\end{array} \\
& \begin{array}{cccc|c}
\nu=3: & \frac{5}{36} & \frac{2}{9}-\frac{\sqrt{15}}{15} & \frac{5}{36}-\frac{\sqrt{15}}{30} & \frac{1}{2}-\frac{\sqrt{15}}{10} \\
\frac{5}{36}+\frac{\sqrt{15}}{24} & \frac{2}{9} & \frac{5}{36}-\frac{\sqrt{15}}{24} & \frac{1}{2} \\
\hline \frac{5}{36}+\frac{\sqrt{15}}{30} & \frac{2}{9}+\frac{\sqrt{15}}{15} & \frac{5}{36} & \frac{1}{2}+\frac{\sqrt{15}}{10} \\
\hline \frac{5}{18} & \frac{4}{9} & \frac{5}{18} &
\end{array}
\end{aligned}
$$

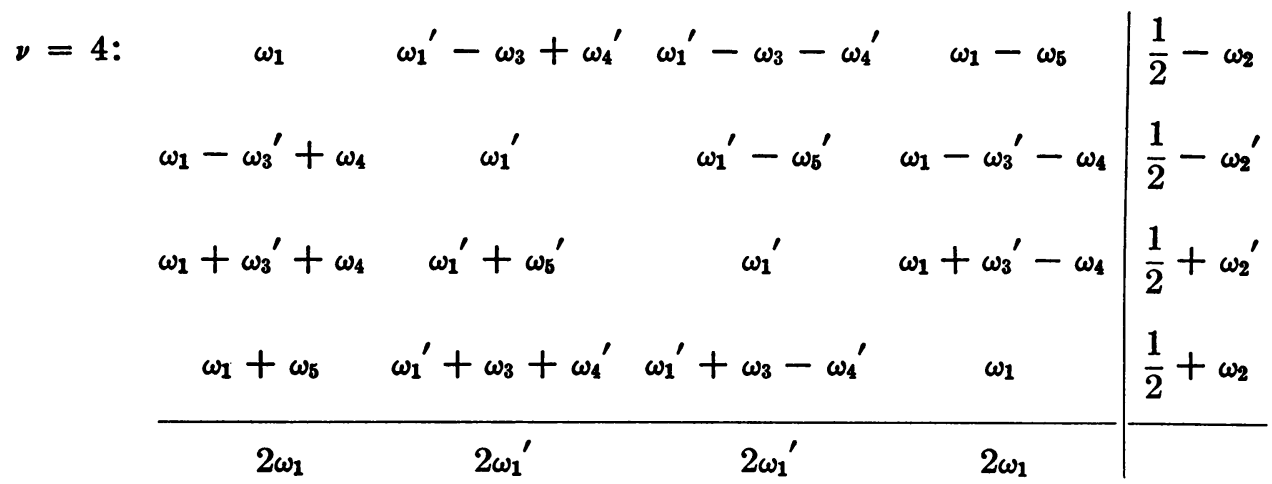

$$
\begin{aligned}
& {\left[\omega_{1}=\frac{1}{8}-\frac{\sqrt{30}}{144}, \quad \omega_{1}^{\prime}=\frac{1}{8}+\frac{\sqrt{30}}{144}, \quad \omega_{2}=\frac{1}{2} \sqrt{\frac{15+2 \sqrt{30}}{35}},\right.} \\
& \omega_{2}^{\prime}=\frac{1}{2} \sqrt{\frac{15-2 \sqrt{30}}{35}}, \quad \omega_{3}=\omega_{2}\left(\frac{1}{6}+\frac{\sqrt{30}}{24}\right), \quad \omega_{3}^{\prime}=\omega_{2}^{\prime}\left(\frac{1}{6}-\frac{\sqrt{30}}{24}\right), \\
& \omega_{4}=\omega_{2}\left(\frac{1}{21}+\frac{5 \sqrt{30}}{168}\right), \quad \omega_{4}^{\prime}=\omega_{2}^{\prime}\left(\frac{1}{21}-\frac{5 \sqrt{30}}{168}\right), \quad \omega_{5}=\omega_{2}-2 \omega_{3}, \\
& \left.\omega_{5}^{\prime}=\omega_{2}^{\prime}-2 \omega_{3}^{\prime}\right] .
\end{aligned}
$$


TABLE 2.-Continued

$$
\nu=5:
$$

$$
\begin{aligned}
& \omega_{1} \\
& \omega_{1}^{\prime}-\omega_{3}+\omega_{4}^{\prime} \\
& \frac{32}{225}-\omega_{5} \omega_{1}^{\prime}-\omega_{3}-\omega_{4}^{\prime} \\
& \begin{array}{l|l}
\omega_{1}-\omega_{6} & \frac{1}{2}-\omega_{2}
\end{array} \\
& \omega_{1}-\omega_{3}^{\prime}+\omega_{4} \\
& \omega_{1}^{\prime} \\
& \frac{32}{225}-\omega_{5}^{\prime} \\
& \omega_{1}^{\prime}-\omega_{6}^{\prime} \\
& \omega_{1}-\omega_{3}^{\prime}-\omega_{4} \\
& \frac{1}{2}-\omega_{2}^{\prime} \\
& \omega_{1}+\omega_{7} \\
& \omega_{1}^{\prime}+\omega_{7}^{\prime} \\
& \frac{32}{225} \\
& \omega_{1}^{\prime}-\omega_{7}^{\prime} \\
& \omega_{1}-\omega_{7} \\
& \frac{1}{2} \\
& \omega_{1}+\omega_{3}^{\prime}+\omega_{4} \\
& \omega_{1}^{\prime}+\omega_{6}^{\prime} \quad \frac{32}{225}+\omega_{5}^{\prime} \\
& \omega_{1}^{\prime} \\
& \omega_{1}+\omega_{3}^{\prime}-\omega_{4} \\
& \frac{1}{2}+\omega_{2}^{\prime} \\
& \omega_{1}+\omega_{6} \\
& \omega_{1}^{\prime}+\omega_{3}+\omega_{4}^{\prime} \frac{32}{225}+\omega_{5} \\
& \omega_{1}^{\prime}+\omega_{3}-\omega_{4}^{\prime} \\
& \frac{1}{2}+\omega_{2} \\
& 2 \omega_{1} \\
& 2 \omega_{1}^{\prime} \\
& \frac{64}{225} \\
& 2 \omega_{1}^{\prime} \\
& 2 \omega_{1} \\
& {\left[\omega_{1}=\frac{322-13 \sqrt{70}}{3600}, \quad \omega_{1}^{\prime}=\frac{322+13 \sqrt{70}}{3600}, \quad \omega_{2}=\frac{1}{2} \sqrt{\frac{35+2 \sqrt{70}}{63}},\right.} \\
& \omega_{2}^{\prime}=\frac{1}{2} \sqrt{\frac{35-2 \sqrt{70}}{63}}, \quad \omega_{3}=\omega_{2}\left(\frac{452+59 \sqrt{70}}{3240}\right), \quad \omega_{3}^{\prime}=\omega_{2}^{\prime}\left(\frac{452-59 \sqrt{70}}{3240}\right), \\
& \omega_{4}=\omega_{2}\left(\frac{64+11 \sqrt{70}}{1080}\right), \quad \omega_{4}^{\prime}=\omega_{2}^{\prime}\left(\frac{64-11 \sqrt{70}}{1080}\right), \quad \omega_{5}=8 \omega_{2}\left(\frac{23-\sqrt{70}}{405}\right), \\
& \omega_{5}^{\prime}=8 \omega_{2}^{\prime}\left(\frac{23+\sqrt{70}}{405}\right), \quad \omega_{6}=\omega_{2}-2 \omega_{3}-\omega_{5}, \quad \omega_{6}^{\prime}=\omega_{2}^{\prime}-2 \omega_{3}^{\prime}-\omega_{5}^{\prime}, \\
& \left.\omega_{7}=\omega_{2}\left(\frac{308-23 \sqrt{70}}{960}\right), \quad \omega_{7}^{\prime}=\omega_{2}^{\prime}\left(\frac{308+23 \sqrt{70}}{960}\right)\right] .
\end{aligned}
$$

Theorem 8. If $A(2 \nu)$ then $B(2 \nu), C(\nu), D(\nu)$.

TheOREM 9. If $B(2 \nu)$ (hence a Gauss-Legendre formula) then either of $C(\nu)$, $D(\nu)$ implies the other and either implies $A(2 \nu)$.

TheOREM 10. Given values of $b_{1}, b_{2}, \cdots, b_{\nu}, c_{1}, c_{2}, \cdots, c_{\nu}$ either $C(\nu)$ or $D(\nu)$ defines $a_{i j}(i, j=1,2, \cdots, \nu)$ uniquely.

To prove Theorem 8 we use Theorems 1 and 2 to deduce $B(2 \nu)$ and $E(\nu, \nu)$ and then Theorems 5 and 6 to deduce $C(\nu)$ and $D(\nu)$.

Theorem 9 also follows easily from the previous results. From Theorems 3 and 4, $B(2 \nu)$ with either of $C(\nu), D(\nu)$ implies $E(\nu, \nu)$; whereas from Theorems 5 and $6, B(2 \nu)$ and $E(\nu, \nu)$ imply both $C(\nu)$ and $D(\nu)$. Finally we use Theorem 7 with the values $\eta=\xi=\nu, \zeta=2 \nu$ to deduce $A(2 \nu)$.

Theorem 10 follows from the fact that $C(\nu), D(\nu)$ each consist of $\nu$ sets of $\nu$ equations in $\nu$ unknowns and since $b_{1}, b_{2}, \cdots, b_{\nu}$ are all non-zero and $c_{1}, c_{2}, \cdots, c_{\nu}$ are all distinct, the Matrices of the coefficients in the various sets are non-singular.

The sets of equations symbolized by $C(\nu)$ are slightly simpler than those symbolized by $D(\nu)$ so it is the former sets that we use for practical evaluations of 
$a_{11}, a_{12}, \cdots, a_{v n}$. To find the parameters in a $\nu$ stage Runge-Kutta process of order $2 \nu$ we may thus perform the following steps

(1) Evaluate the roots of $P_{\nu}(2 c-1)=0$

(2) For each value of $i(i=1,2, \cdots, \nu)$ find $a_{i j}(j=1,2, \cdots, \nu)$ as solutions to the linear system

$$
\sum_{j=1}^{\nu} a_{i j} c_{j}^{k-1}=\frac{1}{k} c_{i}^{k} \quad(k=1,2, \cdots, \nu) .
$$

(3) Find $b_{j}(j=1,2, \cdots, \nu)$ as solutions to the linear system

$$
\sum_{j=1}^{\nu} b_{j} c_{j}^{k-1}=\frac{1}{k} \quad(k=1,2, \cdots, \nu) .
$$

In Table 2 values of the parameters are given for $\nu=1,2,3,4,5$. It will be noticed that for the case $\nu=2$ the process is the same as one suggested by Hammer and Hollingsworth [11].

4. The Error Term. For a process of order $\rho$, the two series (1) and (2) are identical for $r \leqq \rho$. Hence the error vector $\hat{\mathbf{y}}-\mathrm{y}$ is given by

$$
\hat{\mathrm{y}}-\mathrm{y}=\sum_{r>\rho}\left(\beta \Phi \frac{h^{r}}{(r-1) !}-\alpha \frac{h^{r}}{r !}\right) \mathbf{F}=\sum_{r>\rho} \frac{\beta h^{r}}{(r-1) !}\left(\Phi-\frac{1}{\gamma}\right) \mathbf{F}
$$

and we shall suppose that $h$ is sufficiently small for this to be approximated by

where

$$
\sum_{r=\rho+1} \frac{\beta h^{r}}{(r-1) !}\left(\Phi-\frac{1}{\gamma}\right) \mathrm{F}=\frac{h^{\rho+1}}{\rho !} \sum_{r=\rho+1} \beta \delta \mathrm{F}=h^{\rho+1} \sum_{r=\rho+1} \epsilon \mathrm{F}
$$

$$
\delta=\Phi-\frac{1}{\gamma}, \quad \epsilon=\frac{\beta \delta}{\rho !} .
$$

The coefficient of $h^{\rho+1}$ in $\hat{y}-y$ is called by Henrici [12] "the principal error function" and our approximation is to assume that the principal error function is the only important contributor to the truncation error.

We now restrict ourselves to the processes considered in the previous section so that $\rho=2 \nu$. We shall study properties of $\delta$ for the different $F$ of order $2 \nu+1$ so that a simple procedure can be found for evaluating $\epsilon$ in these cases.

Suppose for an elementary differential $\mathbf{F}$ of order $2 \nu+1$ we have

$$
\mathbf{F}=\left\{\mathrm{F}_{1} \mathrm{~F}_{2} \cdots \mathrm{F}_{s}\right\}
$$

and the orders of $F_{1}, F_{2}, \cdots, F_{s}$ are $r_{1}, r_{2}, \cdots, r_{8}$. Then since

$$
1+r_{1}+r_{2}+\cdots+r_{s}=2 \nu+1
$$

it is clear that the number of $r_{1}, r_{2}, \cdots, r_{s}$ which exceed $\nu$ is either 0 or 1 . In the former case we describe $\mathrm{F}$ as a "central" elementary differential and in the latter case a "non-central" elementary differential.

If $\mathrm{F}$ is non-central, suppose $r_{1}>\nu$ and that

$$
\mathbf{F}_{1}=\left\{\overline{\mathbf{F}}_{1} \overline{\mathbf{F}}_{2} \ldots \overline{\mathbf{F}}_{\sigma}\right\} \text {. }
$$

Consider

$$
\mathbf{F}^{\prime}=\left\{\bar{F}_{1} \bar{F}_{2} \cdots \overline{\mathbf{F}}_{\sigma}\left\{\mathbf{F}_{2} \mathbf{F}_{3} \cdots \mathbf{F}_{8}\right\}\right\}
$$


so that the orders $\bar{r}_{1}, \bar{r}_{2}, \cdots \bar{r}_{\sigma}, 2 \nu+1-r_{1}$ of $\overline{\mathbf{F}}_{1}, \overline{\mathbf{F}}_{2}, \cdots, \overline{\mathbf{F}}_{\sigma},\left\{\mathbf{F}_{2} \mathbf{F}_{3} \cdots \mathbf{F}_{s}\right\}$ are each less than $r_{1}$ while the order of $F^{\prime}$ is the same as that of $F$.

Either $F^{\prime}$ is central or we may form $F^{\prime \prime}$ from $F^{\prime}$ in the same way as $F^{\prime}$ was formed from $F$. We may continue this process, forming in turn $F, F^{\prime}, F^{\prime \prime}, \cdots$ until the sequence is terminated at a central elementary differential $\mathbf{F}_{*}$ say. It will be proved that $\delta=-\delta^{\prime}$ and hence that $\delta=-\delta^{\prime}=\delta^{\prime \prime}=\cdots= \pm \delta_{*}$ where $\delta_{*}$ corresponds to $\mathrm{F}_{*}$ and the sign to be used is determined by the parity of the number of members in the sequence $\mathbf{F}^{\prime}, \mathbf{F}^{\prime \prime}, \cdots, \mathbf{F}_{*}$.

We now summarize the principal result of this section.

Theorem 11. If $\mathrm{F}=\left\{\mathrm{f}^{2 \nu}\right\}, \delta=-(\nu !)^{4} /\{(2 \nu) !(2 \nu+1) !\}$.

Theorem 12. If $\mathrm{F}$ is central, $\delta=-(\nu !)^{4} /\left\{[(2 \nu) !]^{2} \gamma\right\}$.

Theorem 13. If $\mathbf{F}$ is non-central, and $\mathbf{F}^{\prime}$ is as defined by (12), $\delta=-\delta^{\prime}$.

To prove Theorem 11 we write the polynomial $P_{\nu}(2 c-1)$ as

$$
P_{\nu}(2 c-1)=p_{0}+p_{1} c+p_{2} c^{2}+\cdots+p_{\nu} c^{\nu},
$$

and consider the equations $B(2 \nu)$,

$$
\sum_{i=1}^{\nu} b_{i} c_{i}^{k-1}=\frac{1}{k}, \quad k=1,2, \cdots, 2 \nu
$$

together with the equation

$$
\sum_{i=1}^{\nu} b_{i} c_{i}^{2 \nu}=\frac{1}{2 \nu+1}+\delta
$$

Multiplying the equations of $B(2 \nu)$ for $k=1,2, \cdots, \nu+1$ by $p_{0}, p_{1}, \cdots, p_{\nu}$ and adding we find

$$
p_{0}+p_{1} / 2+p_{2} / 3+\cdots+p_{\nu} /(\nu+1)=0
$$

and similarly using the values $k=2,3, \cdots, \nu+2$ in the same way and so on we find

$$
\begin{aligned}
& p_{0} / 2+p_{1} / 3+p_{2} / 4+\cdots+p_{\nu} /(\nu+2)=0 \\
& p_{0} / 3+p_{1} / 4+p_{2} / 5+\cdots+p_{\nu} /(\nu+3)=0 \\
& p_{0} /(\nu+1)+p_{1} /(\nu+2)+p_{2} /(\nu+3)+\cdots+p_{\nu}\left[\frac{1}{2 \nu+1}+\delta\right]=0 .
\end{aligned}
$$

We now eliminate $p_{0}, p_{1}, \cdots, p_{\nu}$ from this set of homogeneous linear equations and find

$$
\left|\begin{array}{ccccc}
1 & \frac{1}{2} & \frac{1}{3} & \cdots & \frac{1}{\nu+1} \\
\frac{1}{2} & \frac{1}{3} & \frac{1}{4} & \cdots & \frac{1}{\nu+2} \\
\vdots & \vdots & \vdots & & \vdots \\
\frac{1}{\nu+1} & \frac{1}{\nu+2} & \frac{1}{\nu+3} & \cdots & \frac{1}{2 \nu+1}+\delta
\end{array}\right|=0
$$


so that

$$
\delta=-D_{v+1} / D_{\nu}=-\frac{(\nu !)^{4}}{(2 v) !(2 \nu+1) !}
$$

where we have written (see for example [13])

$$
D_{N}=\left|\begin{array}{ccccc}
1 & \frac{1}{2} & \frac{1}{3} & \cdots & \frac{1}{N} \\
\frac{1}{2} & \frac{1}{3} & \frac{1}{4} & \cdots & \frac{1}{N+1} \\
\vdots & \vdots & \vdots & & \vdots \\
\frac{1}{N} & \frac{1}{N+1} & \frac{1}{N+2} & \cdots & \frac{1}{2 N-1}
\end{array}\right|=\frac{[1 ! 2 ! 3 ! \cdots(N-1) !]^{3}}{N !(N+1) ! \cdots(2 N-1) !} .
$$

This completes the proof of Theorem 11 .

Since $r_{1}, r_{2}, \cdots, r_{s}$ are no more than $v$ for a central elementary differential (8) holds and the proof of the lemma that was introduced before that of Theorem 7 holds in this case as well. We thus have

$$
\gamma \Phi=(2 \nu+1)\left[\phi^{2 \nu}\right]=1-(\nu !)^{4} /[(2 \nu) !]^{2}
$$

and Theorem 12 follows on dividing by $\gamma$.

Using the notation of (10), (11) and (12) we compute using, as usual, equation (31) of [1]

$$
\begin{aligned}
\gamma_{1} & =r_{1} \bar{\gamma}_{1} \bar{\gamma}_{2} \cdots \bar{\gamma}_{\sigma} \\
\gamma & =r_{1}(2 \nu+1) \bar{\gamma}_{1} \bar{\gamma}_{2} \cdots \bar{\gamma}_{\sigma} \gamma_{2} \gamma_{3} \cdots \gamma_{\diamond} \\
\gamma^{\prime} & =(2 \nu+1)\left(2 \nu-r_{1}+1\right) \bar{\gamma}_{1} \bar{\gamma}_{2} \cdots \bar{\gamma}_{\sigma} \gamma_{2} \gamma_{3} \cdots \gamma_{\diamond}
\end{aligned}
$$

so that

$$
\frac{1}{\gamma}+\frac{1}{\gamma^{\prime}}=\frac{1}{\left(2 \nu-r_{1}+1\right) \gamma_{1} \gamma_{2} \cdots \gamma_{s}} .
$$

Again employing the methods of Theorem 7 we arrive at the results

$$
\begin{aligned}
\Phi & =\frac{1}{\gamma_{2} \gamma_{3} \cdots \gamma_{s}}\left[\Phi_{1} \phi^{2 \nu-r_{1}}\right] \\
& =\frac{1}{\left(2 \nu-\gamma_{1}+1\right) \gamma_{2} \gamma_{3} \cdots \gamma_{s}}\left(\Phi_{1}-\left[\bar{\Phi}_{1} \bar{\Phi}_{2} \cdots \bar{\Phi}_{\sigma} \phi^{2 \nu-r_{1}+1}\right]\right) \\
\Phi^{\prime} & =\frac{1}{\gamma_{2} \gamma_{3} \cdots \gamma_{s}}\left[\bar{\Phi}_{1} \bar{\Phi}_{2} \cdots \bar{\Phi}_{\sigma}\left[\phi^{2 \nu-r_{1}}\right]\right] \\
& =\frac{1}{\left(2 \nu-r_{1}+1\right) \gamma_{2} \gamma_{3} \cdots \gamma_{s}}\left[\bar{\Phi}_{1} \bar{\Phi}_{2} \cdots \bar{\Phi}_{s} \phi^{2 \nu-r_{1}+1}\right]
\end{aligned}
$$


Hence

$$
\begin{aligned}
\Phi+\Phi^{\prime} & =\frac{1}{\left(2 \nu-\gamma_{1}+1\right) \gamma_{2} \gamma_{3} \cdots \gamma_{s}} \Phi_{1} \\
& =\frac{1}{\gamma}+\frac{1}{\gamma^{\prime}}
\end{aligned}
$$

which is equivalent to the result of Theorem 13.

Although in principal these results enable us to write down the coefficients for each elementary differential of order $2 \nu+1$ occuring in $\hat{y}-y$, the practical value of this is lessened by the large numbers of such terms which actually occur. However, as some guide to the truncation error we shall evaluate the coefficients $\epsilon_{1}$ and $\epsilon_{2} \dagger$, say, for $F_{1}=\left\{f^{2 \nu}\right\}$ and $F_{2}=\left\{{ }_{2 v} f\right\}_{2 v}$.

These may be regarded as extreme cases; $F_{1}$ is the only elementary differential of order $2 \nu+1$ which involves derivatives of order $2 \nu$ of the elements of $f(y)$ while $F_{2}$ is the only elementary differential of the same order involving only first derivatives. If $\frac{\partial f}{\partial y}$ is the matrix whose $(i, j)$ element is $\frac{\partial f_{i}}{\partial y_{j}}$ then we can write

$$
\begin{aligned}
& \mathbf{F}_{1}=\sum_{i_{1}=1}^{n} \sum_{i_{2}=1}^{n} \cdots \sum_{i_{2 v}=1}^{n} \frac{\partial^{2 v} \mathbf{f}}{\partial y_{i_{1}} \partial y_{i_{2}} \cdots \partial y_{i_{2 v}}} f_{i_{1}} f_{i_{2}} \cdots f_{i_{2},}, \\
& \mathbf{F}_{2}=\left(\frac{\partial \mathrm{f}}{\partial \mathrm{y}}\right)^{2 v} \mathbf{f} .
\end{aligned}
$$

$\delta_{1}$ is given by Theorem 11 as $-(\nu !)^{4} /\{(2 \nu) !(2 \nu+1) !\}$ while $\beta_{1}$ is given immediately from Theorem 7 of [1] as 1.

Hence, we have

$$
\epsilon_{1}=\frac{\beta_{1} \delta_{1}}{(2 \nu) !}=\frac{-(\nu !)^{4}}{[(2 \nu) !]^{3}(2 \nu+1)}
$$

On the other hand, $F_{2}$ is non-central and the central $\mathbf{F}_{*}$ corresponding to it is $\left\{\{\nu-1\}_{\nu-1}^{2}\right\} \cdot \gamma_{*}$ is $(\nu !)^{2}(2 \nu+1)$ so that

$$
\delta_{2}= \pm \delta_{*}=\frac{\mp(\nu !)^{2}}{(2 \nu) !(2 \nu+1) !}
$$

and the upper (or lower) sign is to be used if $\nu$ is even (or odd). It is easy to see that $\beta_{2}$ is $(2 \nu)$ ! Thus

$$
\begin{aligned}
\epsilon_{2} & =\frac{\beta_{2} \delta_{2}}{(2 \nu) !}=\frac{\mp(\nu !)^{2}}{(2 \nu) !(2 \nu+1) !} \\
& = \pm\left(\begin{array}{c}
2 \nu \\
\nu
\end{array}\right) \epsilon_{1} .
\end{aligned}
$$

The first few values of $\epsilon_{1}$ and $\epsilon_{2}$ may be found from Table 3 .

It is natural to compare the integration processes described here with methods based on quadrature formulae such as the method of Stoller and Morrison [14]. In

$\dagger$ The convention concerning subscripted constants $\alpha, \beta, \gamma$ will be extended to $\delta$ and $\epsilon$. 
TABLE 3

\begin{tabular}{|c|c|c|}
\hline$\nu$ & $1 / \epsilon_{1}$ & $1 / \epsilon_{2}$ \\
\hline $\begin{array}{l}1 \\
2 \\
3 \\
4 \\
5\end{array}$ & $\begin{array}{rr} & -24 \\
-2 & 016000 \\
-1778 & 112000 \\
-2 & 534876 \\
467200\end{array}$ & $\begin{array}{r}+12 \\
-720 \\
+100800 \\
-25401600 \\
+10059033600\end{array}$ \\
\hline
\end{tabular}

this type of method $y_{i}$ is estimated at $x_{0}+c_{i} h(i=1,2, \cdots, \nu)$ by some convenient integration process and $\mathrm{y}$ is finally found at $x_{0}+h$ by the quadrature formula

$$
\mathrm{y}=\mathrm{y}_{0}+h \sum_{i=1}^{\nu} b_{i} \mathbf{f}\left(\mathbf{y}_{i}\right)
$$

with an error supposed due only to the quadrature formula.

In the cases where Gauss-Legendre quadrature is used it turns out that the principal error function is

$$
\begin{aligned}
& \epsilon_{1} h^{2 \nu+1}\left(\frac{d}{d x}\right)^{2 \nu+1} \mathrm{y}_{0} \\
= & \epsilon_{1} h^{2 \nu+1}\left(\sum_{r=2 v+1} \alpha \mathbf{F}\right)
\end{aligned}
$$

and no coefficient $\epsilon_{1} \alpha$ exceeds the corresponding $\epsilon$ found for the processes studied in this paper. Although no general rule can be made this suggests that there is a tendency for the methods of this paper to be the less accurate. On the other hand the lack of suitable integration formulae of sufficiently high accuracy for estimating $\mathbf{y}_{i}(i=1,2, \cdots, \nu)$ in the cases $\nu=3,4, \cdots$ is a disadvantage of the other method.

5. A Numerical Example. We now illustrate the process with $\nu=3$ using it to find $y(0.3)$ where

$$
y^{\prime}=y, \quad y(0)=1 \text {. }
$$

For this equation all elementary differentials of order $2 \nu+1=7$ vanish identically except $F_{2}$ so we find a principal error function $y h^{7} / 100800$.

In the vicinity of $y=1$ the value $h=.3$ gives as an estimate of the truncation error $(.3)^{7} / 100800 \approx 22 \times 10^{-10}$.

Using the starting values $g^{(1)}=g^{(2)}=g^{(3)}=1$ and working to $10 D, 8$ iterations are required for convergence. The final values are $g^{(1)}=1.0343925937, g^{(2)}=$ $1.1618293499, g^{(3)}=1.3049861726$ giving $y(0.3)=1.3498588105$. This exceeds the true result, $\exp (0.3)=1.3498588076$ by $29 \times 10^{-10}$ in reasonable agreement with our error estimate.

Appendix. For a non-explicit process, $\mathbf{g}^{(1)}, \mathbf{g}^{(2)}, \cdots, \mathbf{g}^{(\nu)}$ would normally be found by an iterative procedure. If $\mathbf{g}_{N}{ }^{(i)}$ denotes iterate number $N$ for $\mathbf{g}^{(i)}$ then a possible process would be

$$
\mathbf{g}_{N}{ }^{(i)}=\mathbf{f}\left(\mathrm{y}_{0}+h\left(\sum_{j=1}^{i-1} a_{i j} \mathbf{g}_{N}{ }^{(i)}+\sum_{j=i}^{\nu} a_{i j} \mathbf{g}_{N-1}^{(j)}\right)\right) .
$$


We define $l$ as the greatest of

$$
\left|a_{21}\right|,\left|a_{31}\right|+\left|a_{32}\right|, \cdots,\left|a_{n 1}\right|+\left|a_{v 2}\right|+\cdots+\left|a_{v v-1}\right|
$$

$u$ as the greatest of

$$
\left|a_{11}\right|+\left|a_{12}\right|+\cdots+\left|a_{1 v}\right|,\left|a_{22}\right|+\left|a_{23}\right|+\cdots+\left|a_{2 v}\right|, \cdots,\left|a_{v v}\right|
$$

and $a$ as $l+u$. For a vector $\nabla .=\left(v_{1}, v_{2}, \cdots, v_{n}\right)$ we denote by $\|\nabla\|$ the greatest of $\left|v_{1}\right|,\left|v_{2}\right|, \cdots,\left|v_{n}\right|$ (any other norm could be used in the discussion which follows but we choose this one for definiteness). We now prove the following result:

Theorem 14. If $\mathrm{f}(\mathrm{y})$ satisfies a Lipschitz condition,

$$
\|\mathbf{f}(\mathbf{y})-\mathbf{f}(\mathbf{z})\| \leqq L\|\mathbf{y}-\mathbf{z}\|
$$

and $|h|<1 /($ La $)$ then the equations defining $\mathbf{g}^{(1)}, \mathbf{g}^{(2)}, \cdots, \mathbf{g}^{(v)}$ have a unique solution and $\mathbf{g}_{N}{ }^{(1)}, \mathbf{g}_{N}{ }^{(2)}, \cdots, \mathbf{g}_{N}{ }^{(p)}$ defined by (13) tend to this solution as $N$ tends to infinity.

To prove that there is no more than one solution we assume, on the contrary that there are two and denote these by $\mathbf{g}^{(1)}, \mathbf{g}^{(2)}, \cdots, \mathbf{g}^{(v)}$ and $\overline{\mathbf{g}}^{(1)}, \overline{\mathbf{g}}^{(2)}, \cdots, \overline{\mathbf{g}}^{(v)}$ so that

$$
\begin{aligned}
& \mathbf{g}^{(i)}=\mathrm{f}\left(\mathrm{y}_{0}+h \sum_{j=1}^{\nu} a_{i j} \mathbf{g}^{(j)}\right) \\
& \overline{\mathbf{g}}^{(i)}=\mathrm{f}\left(\mathrm{y}_{0}+h \sum_{j=1}^{\nu} a_{i j} \overline{\mathbf{g}}^{(j)}\right)
\end{aligned}
$$

Hence we have

$$
\begin{aligned}
\left\|\mathbf{g}^{(i)}-\overline{\mathbf{g}}^{(i)}\right\| & =\left\|\mathbf{f}\left(\mathrm{y}_{0}+h \sum_{j=1}^{\nu} a_{i j} \mathbf{g}^{(j)}\right)-\mathbf{f}\left(\mathrm{y}_{0}+h \sum_{j=1}^{\nu} a_{i j} \overline{\mathbf{g}}^{(j)}\right)\right\| \\
& \leqq|h| L\left\|\sum_{j=1}^{\nu} a_{i j}\left(\mathbf{g}^{(j)}-\overline{\mathbf{g}}^{(j)}\right)\right\| \\
& \leqq|h| L \sum_{j=1}^{\nu}\left(\left|a_{i j}\right| \cdot\left\|\mathbf{g}^{(j)}-\overline{\mathbf{g}}^{(j)}\right\|\right) \\
& \leqq|h| L a \max _{j}\left\|\mathbf{g}^{(j)}-\overline{\mathbf{g}}^{(j)}\right\| .
\end{aligned}
$$

Hence

$$
\left\|\mathbf{g}^{(i)}-\overline{\mathbf{g}}^{(i)}\right\|<\max _{j}\left\|\mathbf{g}^{(j)}-\overline{\mathbf{g}}^{(j)}\right\|
$$

for all $i$, a contradiction.

To prove the convergence of the iterative scheme a similar calculation to the above shows that

$$
\left\|\mathbf{g}_{N}{ }^{(i)}-\mathbf{g}_{N-1}^{(i)}\right\| \leqq|h| L\left(l \max _{j}\left\|\mathbf{g}_{N}{ }^{(j)}-\mathbf{g}_{N-1}^{(j)}\right\|+u \max _{j}\left\|\mathbf{g}_{N-1}^{(j)}-\mathbf{g}_{N-2}^{(j)}\right\|\right)
$$

and hence that 


$$
\begin{aligned}
\max _{j}\left\|\mathbf{g}_{N}^{(j)}-\mathbf{g}_{N-1}^{(j)}\right\| & \leqq \frac{|h| L u}{1-|h| L l} \max _{j}\left\|\mathbf{g}_{N-1}^{(j)}-\mathbf{g}_{N-2}^{(j)}\right\| \\
& =|h| L\left(a-\frac{a-a|h| L l-u}{1-|h| L l}\right) \max _{j}\left\|\mathbf{g}_{N-1}^{(j)}-\mathbf{g}_{N-2}^{(j)}\right\| \\
& \leqq k \max _{j}\left\|\mathbf{g}_{N-1}^{(j)}-\mathbf{g}_{N-2}^{(j)}\right\|
\end{aligned}
$$

where $k=|h| L a<1$.

From this it easily follows that every element of $\mathbf{g}_{N}^{(i)}-\mathbf{g}_{N-1}^{(i)}$ has modulus less than $M k^{N}$ for some fixed $M$ and this ensures the convergence of the sequence $\mathrm{g}_{N}{ }^{(i)}, N=1,2, \cdots$.

Suppose the limit of the sequence is $\mathbf{g}^{(i)}$. It is trivial to show that $\mathbf{g}^{(1)}, \mathbf{g}^{(2)}, \cdots, \mathbf{g}^{(v)}$ satisfy the equation (14).

Finally it may be noted that the transformation $\mathbf{g}_{N-1}^{(i)} \rightarrow \mathbf{g}_{N}^{(i)}$ is a "contraction mapping" [15] and Theorem 14 is a special case of a fundamental theorem on such mappings in complete metric spaces.

University of Canterbury

Christchurch, New Zealand

1. J. C. BUtcher, "Coefficients for the study of Runge-Kutta integration processes," J. Aust. Math. Soc., v. 3, 1963, p. 185-201.

2. C. RUNGE, "Über die numerische Auflösung von Differential-gleichungen," Math. Ann., v. 46,1895 , p. $167-178$.

3. W. KUTTA, "Beitrag zur naherungsweisen Integration von Differentialgleichungen," Zeit. Math. Physik, v. 46, 1901, p. 435-453.

4. E. J. NYSTRÖM, "Über die numerische Integration von Differentialgleichungen," Acta Soc. Sci. Fennicae, v. 50, 1925, p. 1-55.

5. S. GILL, "A process for the step-by-step integration of differential equations in an automatic digital computing machine," Proc. Cambridge Philos. Soc., v. 47, 1951, p. 96-108.

6. A. HणT̃, "Une amélioration de la méthode de Runge-Kutta-Nyström pour la résolution numérique des équations différentielles du premier ordre," Acta Fac. Nat. Univ. Comenian. Math., v.1, 1956, p. 201-224.

7. A. HUđA, "Contribution à la formule de sixième ordre dans la méthode de RungeKutta-Nyström," Acta Fac. Nat. Univ. Comenian. Math., v. 2, 1957, p. 21-24.

8. K. S. KUNz, Numerical Analysis, 1st edition, McGraw-Hill, New York, 1957, p. 206.

9. R. H. Merson, "An operational method for the study of integration processes," Proceedings of Conference on Data Processing and Automatic Computing Machines at Weapons Research Establishment, Salisbury, South Australia, 1957, paper No. 110.

10. Z. Kopal, Numerical Analysis, 1st edition, Chapman and Hall, London, 1955, p. 368.

11. P. C. HAMmer \& J. W. HollingsworTh, "Trapezoidal methods of approximating solutions of differential equations," $M T A C$ v. 9, 1955, p. 92-96.

12. P. HenricI, Discrete Variable Methods in Ordinary Differential Equations, 1st edition, John Wiley \& Sons, Inc., New York, 1962, p. 131.

13. T. Muir, A Treatise on the Theory of Determinants, Dover, New York, 1960, p. 437.

14. L. Stoller \& D. Morrison, "A method for the numerical integration of ordinary differential equations," MTAC., v. 12, 1958, p. 269-272.

15. A. N. Kolmogorov \& S. V. Fomin, Elements of the Theory of Functions and Functional Analysis, v. 1, Graylock Press, Rochester, 1957, p. 43. 